\title{
Authoring and E-LOTOS conception of interactive networked multimedia applications in MUSE environment
}

\author{
L. P. Gaspary and M. J. Almeida \\ Universidade Federal do Rio Grande do Sul \\ Campus do Vale - Bloco IV, Porto Alegre, Brazil \\ Phone: ++55 (51) 316-6161, Fax: ++55 (51) 319-1576 \\ E-mail: \{paschoal, janilce\}@inf.ufrgs.br
}

\begin{abstract}
This work presents MUSE, a graphical environment for modeling interactive networked multimedia applications. Through an advanced graphic interface and a new high-level authoring model, it is possible to create complex systems in a fast and intuitive way. The authoring model proposed in this work and adopted by the tool deals with media objects distributed in a computer network, allowing the definition of acceptable delay thresholds and alternative media objects. Due to the large expressiveness of the model, however, specifications with logical and temporal inconsistencies may be generated. For this reason, the tool also provides E-LOTOS specifications used with the purpose of analyzing and verifying the applications aiming at validating the temporal requirements defined by the author.
\end{abstract}

\section{Keywords}

Multimedia, synchronization, formal conception, E-LOTOS, validation 


\section{INTRODUCTION}

The advance of the utilization of multimedia applications in several fields of human activity is remarkable. Independent of the area, whether education or entertainment, the possibility to aggregate dynamic resources like audio and video to the ones already widely used like text and image results in benefits to the users of such applications. Besides, with the popularization of the Internet, there is an increasing demand for their execution in distributed environments.

The possibility of having an application with its media objects dispersed in a network influences the creation and modeling of such applications. Users must provide the authoring tools with information like temporal restrictions, defining acceptable delay thresholds to the presentation of the elements that compose the system and establishing the presentation of alternative media objects.

The definition of these restrictions is accomplished based on a synchronization model, which dictates the rules about how the media objects of an application can be related in time. Several synchronization models have been proposed (Blakowski, 1996). Most of them are both flexible and very expressive. That is the reason why the resulting specifications can be source of incoherences where the logical and temporal consistency of the involved media objects can not be assured. An alternative would be to use directly a formal description technique (FDT) to describe the applications, making its analysis possible and so guaranteeing its consistency. The disadvantage of this direct use, however, is the high complexity inherent to FDTs. So, the need of having a structured high-level model to specify interactive networked multimedia applications becomes evident. The resulting specifications shall then be translated to a FDT so that verification and simulation methods can be applied to them.

In this context, an interactive networked multimedia applications authoring model was created. MUSE (MUltimedia Applications Specification Environment) was developed to support this model, allowing the user to easily define a multimedia presentation according to the MHEG-5 standard (ISO, 1995). The adoption of MHEG-5 allows multimedia information to be shared without worrying about the platform or operating system used, providing specification and development of portable applications. To make the validation process of the specifications possible, the environment automatically generates E-LOTOS specifications. The results obtained from the validation are presented to the author in a quite readable way in the own environment. After the elimination of the incoherences, MHEG-5 applications are generated and can be executed by a MHEG engine. This work is part of DAMD project (Distributed Multimedia Applications Design), sponsored by the Brazilian research council.

This paper is organized as follows: section 2 presents important aspects to be considered in the applications authoring process, relating them to some multimedia synchronization models pointed by the literature. This section also presents the proposed authoring model. In section 3 basic aspects of E-LOTOS FDT as well as a mechanism to represent in this language, specifications generated by the authoring model are presented. Section 4 illustrates the functionality of the environment and in section 5, one can read the final considerations. 
The specification of multimedia applications is accomplished with base in three fundamental aspects: logical structuring, establishment of temporal relationships and spatial definition among the elements belonging to the application. The logical structuring is concerned to offer abstraction mechanisms, providing a wide and structural view of the application. The specification of the temporal behavior involves the definition of synchronization relations among media objects. The spatial synchronization cares about adjusting the positioning of the visible media objects according to the output devices (video).

The temporal relations are established according to a synchronization model, which imposes rules on how these elements can relate to each other. Several models have been proposed in the literature. One of the most adopted by existent authoring tools is the time-line based one (Hirzalla, 1995). However it presents many limitations such as the difficulty both to modularize the application and to establish relations among elements with variable or unknown duration like user interaction for example (Soares, 1997). Models based on restrictions like HTSPN (Hierarchical Time Stream Petri Nets) (Sénac, 1995) and object-oriented models do not present these problems. On the other hand, these models have not been widely used in commercial tools because of the difficulty in understanding the specifications resulting from them.

In this work, an authoring model that joins mechanisms for logical structuring the applications to a synchronization model similar to HTSPN is proposed. The logical structuring level is based on the concept of scenes and groups, providing a broad view of the application. The definition of temporal synchronizations is done in each scene by means of a simplified graph. The spatial synchronization allows media objects to be positioned considering the output device.

\subsection{Logical structuring}

The complexity of multimedia applications increase according to the growth of involved media objects and, consequently, to the several temporal relationships established among them. This is the fundamental reason why the specification of these applications in only one plane is inappropriate. To solve this problem, the concept of scenes was incorporated into the model considering the MHEG-5 standard. Multimedia applications can be organized as a group of scenes related by events, which provide the navigation among them. Each of these scenes can be seen as a black box with an internal behavior that, under certain conditions, enables the presentation of other scenes. In figure 1, one can see an application structured in three scenes: Scene1, Scene2 and Scene3.

The use of this concept, however, does not solve the problem of complexity at all, since a specification with many scenes will be hardly understood. Trying to make the understanding of so large applications easier, a hierarchy mechanism was added to the model by means of the concept of group of scenes. 


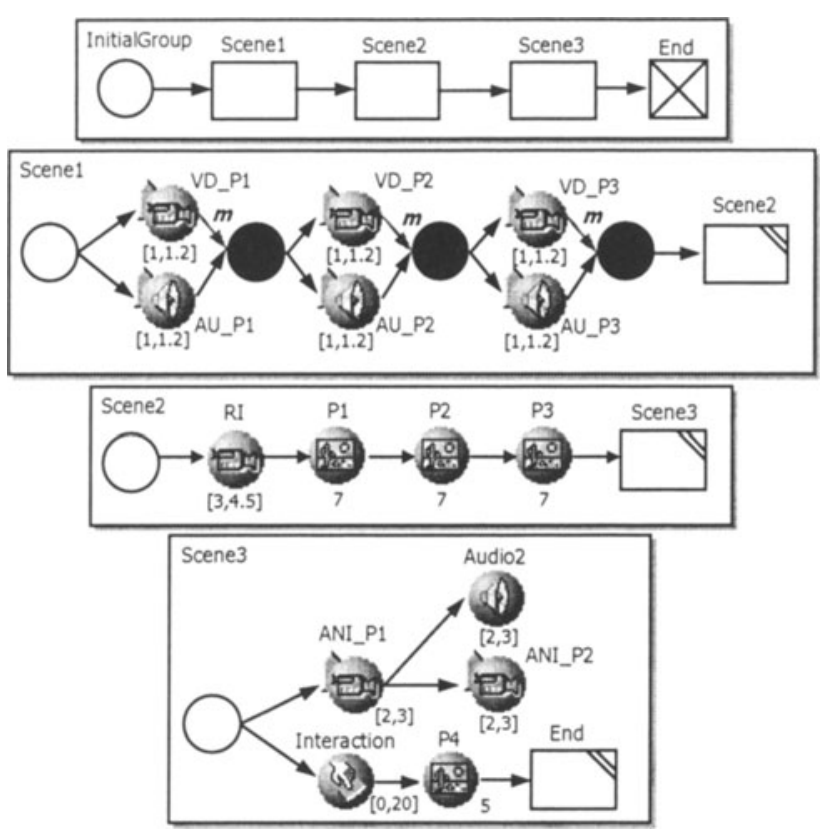

Figure 1 Representation of a simple example.

\subsection{Temporal synchronization}

The temporal synchronization of an application, as mentioned previously, refers to the ordering of the presentation of its media objects in time. Each media object has a presentation duration that may or may not be foreseen, depending on its nature. The following topics present how the synchronization relationships can be established.

\section{Basic synchronization}

Media objects can be presented sequentially or simultaneously. In the sequential presentation, the playout of a media object depends on the end of the presentation of another media object. Scene2 (see figure 1) models the sequential presentation of a recorded interaction (RI) and three images (P1, P2 and P3). The parallelism, on the other hand, considers that media objects start to be presented from a same instant. This is modeled in Scene3, where the animation fragment ANI_P1 is presented in parallel with a button (Interaction).

Duration of the presentation of media objects and acceptable delay thresholds A minimum and a maximum duration of presentation are associated to each media object. In the case of an image or a text, these values are equivalent because they are time-independent media objects. When one deal with media objects like audio and video, however, it is important to determine both a minimum and a maximum 
presentation duration, since these media objects will be hardly presented at the nominal rate due to problems like network traffic (see figure 1). The representation of these durations is given by an interval.

\section{Interaction mechanisms and scene transition}

User interaction corresponds, for instance, to a button click or an object selection. It is represented in this model as a constructor whose presentation duration is uncertain, varying between the minimum and maximum values associated to it. When the maximum threshold is reached, the scene continues with its presentation. It is still possible to specify a button without maximum duration; in this case, its evolution will only happen after the interaction. Scene3 (figure 1) shows a button (Interaction) with duration of $[0,20]$. It means that the user will have 20 seconds to select it. When this time elapses, $\mathrm{P} 4$ will be presented.

The user interference is normally associated to a scene transition. Transition is the constructor that makes the navigation among scenes possible. Its execution involves both the immediate suspension of the presentation of all the media objects belonging to the current scene and the beginning of a new scene presentation. In Scene1 (see figure 1), one can see the transition to Scene2. The transitions are also modeled in both Scene2 and Scene3.

\section{Synchronization points}

Synchronization points allow the beginning of the presentation of one or more media objects to be associated to different policies related to the end of the presentation of other media objects that converge for these points. To improve the specification power, the model has adopted some widely commented firing rules in the literature. They allow the association of different behaviors to the synchronization points (Sénac, 1994). The rules supported by the model are the following:

- Master: a synchronization point is fired when the presentation of a master media object is finished, interrupting all the other ones. The master media object is identified by the presence of the character $m$ or the word master close to it. This is the rule adopted by the synchronization points of Scene1 (figure 1).

- Earliest: the synchronization point is fired when the presentation of the first media object is finished, interrupting all the media objects that are running simultaneously. Graphically, this policy is represented by the presence of the character e or the word earliest close to the synchronization point.

- Latest: the absence of an indication close to the media object or to the synchronization point means that all the media objects that precede this point will be executed (or they will conclude due to the elapsing of their maximum presentation duration) before the synchronization point is fired.

\section{Synchronization instants}

In MUSE, the synchronization among components in other instants than the beginning and end of their presentations requires the division of these components in parts, creating a group of segments. The granularity of this division is directly 
associated to the precision degree wished for the synchronization. Scenel (figure 1) shows such synchronization between a video (VD) and an audio (AU). Both of them were fragmented in three segments.

\subsection{Spatial synchronization}

The spatial synchronization allows the author to organize the positioning of the visible components of a scene. It is not possible to accomplish the spatial synchronization considering a certain elapsed time after the beginning of the scene presentation. It is so, because in each execution, due to the acceptable temporal variations, the components can be presented in different instants. For this reason, the spatial synchronization is always accomplished with base in the presentation of a component. The spatial disposition of the components of Scene2 (figure 1) during the presentation of $\mathrm{P} 1$, for example, will allow to organize only the component P1. If in the definition of this scene there were other components defined to be simultaneously presented with $\mathrm{P} 1$, these components would also appear in this view.

\section{REPRESENTING MULTIMEDIA APPLICATIONS IN E-LOTOS}

The formalization of specifications is important for the process of their validation. The proposed authoring model, due to its high flexibility and expressiveness, allows both temporally and logically incoherent specifications to be defined. The analysis process detects, for example, conflicts in resources usage and tests if the application end can be reached from all the possible navigation paths. Thus, specifications described by an author according to the model presented in the previous section are translated to a formal representation, analyzed and the obtained results are presented to the user, who will make the necessary adjustments.

The formal description technique E-LOTOS (Enhancements to LOTOS) (ISO, 1997) is an enhanced version of LOTOS and is in standardization process. The main innovation of the language is the incorporation of quantitative time notion, allowing the definition of instants in which actions or events may happen. This is a fundamental feature for representing multimedia applications and for this reason, E-LOTOS was chosen to formally represent them.

The representation of multimedia applications is hierarchical and considers the four essential elements of the authoring model: application, group, scene and media object. All these elements are modeled as processes that evolve according to previously established synchronization relationships. The way of formally represent multimedia applications commented in this section is based on the approach presented in (Courtiat, 1996). Further details are presented in the following topics. 


\subsection{Data representation and root process instantiation}

Data representation is done by means of a library called classes, which define data types for all possible media objects. There are types like BitmapClass, StreamClass and SwitchButtonClass, whose definition is based on their respective MHEG-5 classes. For example, the fields of BitmapClass are the media object, its position in the output device and its dimensions. The application is started from the instantiation of the root group process (InitialGroup). After that, the application is indeed able to evolve.

\subsection{Group representation}

In the representation of groups, the hiding operator is used. Taking the example of figure 2, one can see that some internal events like the beginning of both Scene2 (s_Scene2) and Scene3 (s_Scene3) are not visible outside the process (1).
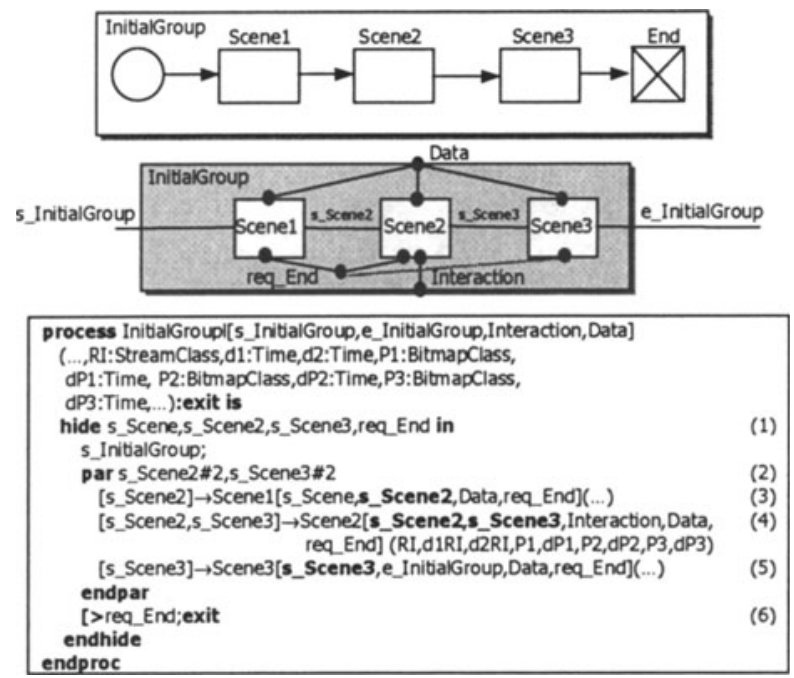

Figure 2 InitialGroup modeling in E-LOTOS.

These events are used to synchronize the presentation of the scenes belonging to InitialGroup. The synchronization is modeled with the par operator (2). For instance, the beginning of Scene2 is associated with the end of Scene1 (s_Scene2) (3 and 4). The same occurs with Scene2 and Scene3: the beginning of the later is synchronized with the end of Scene2 (s_Scene3) (4 and 5).

The disabling operator must also be mentioned (6). As one can observe, the req_End event reaches all the processes of the group; it is used to model the end of the application. When generated (by a transition to end), groups and scenes are terminated with exit. 


\subsection{Scene representation}

Scene modeling differs in many aspects from group representation. One of the differences is that scene processes instantiate media objects and restrictions instead of groups and scenes. The presence of the loop operator in the representation is another important difference (1). It is used to allow a scene to be presented more than once, which may happen when the application is logically organized as a net. Figure 3 shows Scene2 previously instantiated in figure 2 .

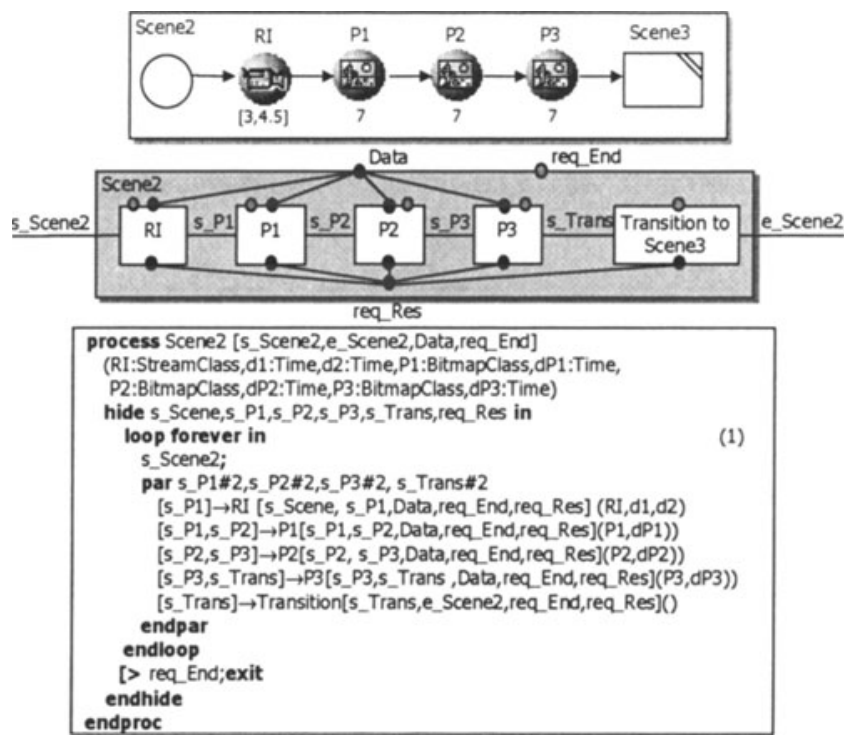

Figure 3 Representation of Scene2.

The req_Res event is responsible for restarting the media objects of the current scene when a transition to another scene occurs. The code that models a scene transition is composed of three events: s_Trans, req_Res and f_Scene (see figure 4a). The former denotes the occurrence of the transition. The second invokes the media objects of the scene to be reset. The third one indicates the end of the scene presentation. As the transition is a never end process, it is also disabled by the occurrence of the req_End event. When the transition is to the end of the application, the req_Res event is replaced by the req_End event (see figure 4b).

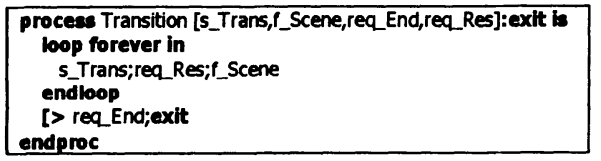

(a) Scene transition

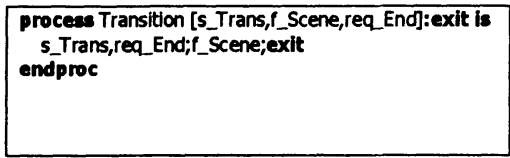

(b) Transition to the end of the application

Figure 4 Representation of transitions. 


\subsection{Basic objects and temporal restrictions}

Basic or monolithic objects were defined by (Hirzalla,1995) and model the presentation of simple media objects. These media objects are defined by the occurrence of synchronous (beginning, end) and asynchronous (user interaction) events. Several combinations of these events can be formulated, but only eight are pointed as important in the definition of interactive multimedia scenes. This work presents three of these combinations (see table 1). The fourth object presented in this table (pSsSe - Synchronous start Synchronous end) doesn't appear in (Hirzalla,1995). It allows time-dependent media with both minimum and maximum presentation durations to be modeled. In the definition of the processes, the Data event was used to represent the presentation of the media object.

Table 1 Representation of basic objects

\begin{tabular}{|c|c|}
\hline E-LOTOS Code & Description \\
\hline \multicolumn{2}{|l|}{ Synchronous start Synchronous end } \\
\hline $\begin{array}{l}\text { process pSsSe[start,end,Data:class] } \\
\text { (media:class,d:time):exit is } \\
\text { start;Data(!media);wait(d); end@t[t=0]; exit } \\
\text { endproc }\end{array}$ & $\begin{array}{l}\text { Used to model time-independent media } \\
\text { objects like image and text with a } \\
\text { presentation known duration. }\end{array}$ \\
\hline \multicolumn{2}{|l|}{ Synchronous start Asynchronous maximum end } \\
\hline $\begin{array}{l}\text { process pSsAme[start,end, user,Data:class] } \\
\text { (media:class,d1,d2:time):exit is } \\
\text { start; Data(!media);wait(d1); } \\
\text { (user@t[t<=d2] [] wait(d2); exit);end@t[t=0];exit } \\
\text { endproc }\end{array}$ & $\begin{array}{l}\text { Used to model user interaction; if the } \\
\text { interaction doesn't occur during the } \\
\text { interval }[\mathrm{d} 1, \mathrm{~d} 2] \text { the process is finished } \\
\text { when the maximum time }(\mathrm{d} 2) \text { is reached. }\end{array}$ \\
\hline \multicolumn{2}{|l|}{ Synchronous start Asynchronous end } \\
\hline $\begin{array}{l}\text { process pSsAe[start,end, user,Data:class] } \\
\text { (media:class,d:time): : exit is } \\
\text { start; Data(!media);wait(d); user; end@t[t=0]; exit } \\
\text { endproc }\end{array}$ & $\begin{array}{l}\text { Modeling of user interaction without a } \\
\text { maximum time to wait defined. The } \\
\text { process finishes only once the interaction } \\
\text { occurs. }\end{array}$ \\
\hline \multicolumn{2}{|l|}{ Synchronous start Synchronous maximum end } \\
\hline $\begin{array}{l}\text { process pSsSme[start,end,Data:class] } \\
\text { (media:class,d1,d2:time) : exit is } \\
\text { start;Data(!media);wait(d1); end@t[t<d2];exit } \\
\text { endproc }\end{array}$ & $\begin{array}{l}\text { Used to model time-dependent media } \\
\text { objects like audio and video, which have a } \\
\text { minimum and a maximum duration } \\
\text { defined. }\end{array}$ \\
\hline
\end{tabular}

Figure 5 shows the representation of P2, which appeared in the definition of Scene 2 in figure 3. The event req_End (3) can again be observed, because media objects are also always being executed (1); if there is a loop in the scene definition, some media objects may be executed more than once during the presentation of the scene. In the same figure, one can also see the effect of the occurrence of the req_Res event: the restart of the media object to its initial state (2).

The authoring model and consequently the tool, to be described in the next section, provide the definition of three distinct temporal restrictions: WaitMaster, WaitLatest and WaitEarliest. Their E-LOTOS representation controls the end of the media objects that converge to the synchronization point. Restrictions are not implemented in libraries because their behaviour depends on the number of media objects that converges to the synchronization point. Figure 6 shows the representation of the WaitEarliest restriction. 


\begin{tabular}{|l|}
\hline process $P 2$ [s_P2,e_P2, Data,req_End,req_Res]:exit is \\
(P2:BitmapClass,dP2:Time) \\
loop forever in \\
pSsSe[s_P2, e_P2, Data] (P2,dP2) \\
[>req_Res \\
$\begin{array}{l}\text { endloop } \\
\text { [> req_End;exit } \\
\text { endproc }\end{array}$ \\
\hline
\end{tabular}

Figure 5 Representation of P2 media object.

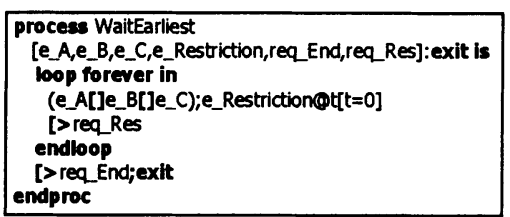

Figure 6 WaitEarliest restriction.

\section{THE AUTHORING ENVIRONMENT}

The authoring environment is divided in two units: media repository and specification area. At any moment, the user may insert media objects into the repository. This is done by browsing a local media object or referencing a remote one. The specification area is composed of the scenes and groups of an application. Each scene is represented by two different views: the temporal and the spatial one. The temporal view allows the user to insert icons and to establish their relationship using arches. The visible elements, used in the temporal synchronization, can be adjusted in the spatial view.

Figure 7 shows the basic functionality of the authoring environment. The toolbar has shortcuts to its main functions (1). Two support windows can be observed: Specification Hierarchy (2) and Icons Palette (3). The former provides the user a general view of the application, presenting all the scenes and groups in a tree and providing a structured view of the relationships among them. In this case, the modeled application was the example presented in section 2 and is composed, therefore, of three scenes: Scene1, Scene2 and Scene3 (4). The later, in its turn, provides mechanisms to visualize and edit the properties of the icons. In the same figure, the bitmap icon (P1) of Scene2 is selected and its specific properties are presented in the mentioned window. Icons that have an associated media object (audio, text, image, and video) present a special property called media. This property must be filled with a media object existing in the repository. In this example, icon P1 is associated to the media object Rio Bonito.

In figure 7, one can also observe the specification of Scene2 (5). It is composed of video (RI) followed by the sequential presentation of three images (P1, P2 and $\mathrm{P} 3)$. By the end of the presentation of the last media object, a transition to Scene3 occurs. These information are presented by the temporal view. At the same time, the spatial view of Scene2 taking the icon P1 as reference is showed (6). It is possible to move or resize the visible media objects. Their properties related to coordinates and dimensions are automatically updated. 


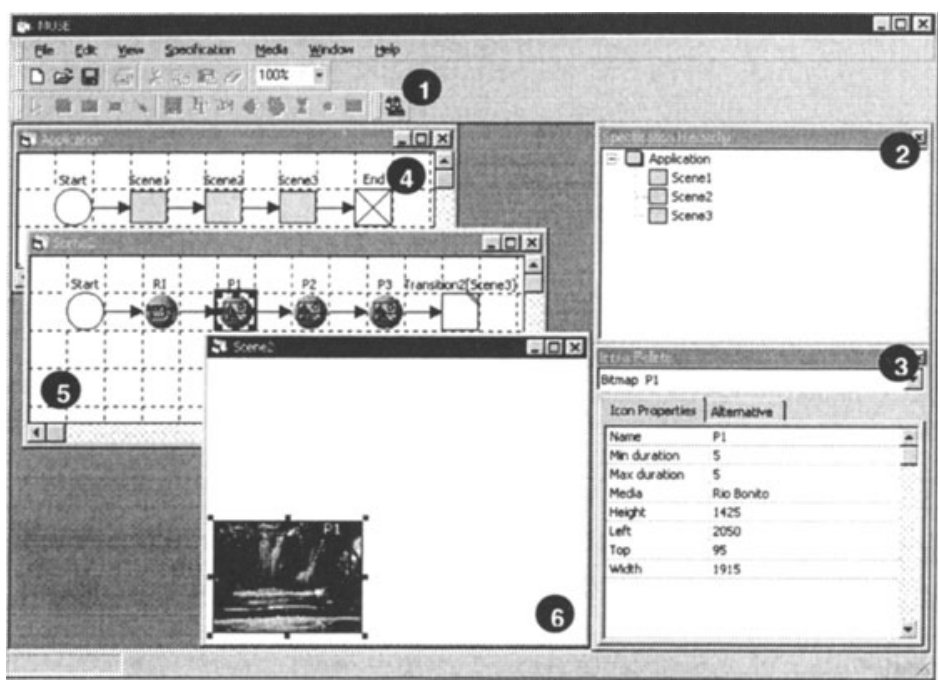

Figure 7 Graphic interface of the authoring environment.

Time-dependent media objects, like video, can be divided in smaller segments, allowing the synchronization of other media objects with specific points of them. The environment provides mechanisms that make the process of fragmentation of these media objects easy. MUSE also provides means to reuse scenes and groups. It can be done by browsing the group or scene to be retrieved. It is necessary just to redefine the transitions, defining where the application should evolve to after its presentation. Finally, it is also important to highlight the functionality of E-LOTOS code generation. This is obtained through the special saving option Save as ELOTOS.

\section{CONCLUSIONS}

This work initially proposed a new model for specifying interactive networked multimedia application. Besides, mechanisms for mapping this model to the ELOTOS language were presented. Finally, the developed environment was described. The main contribution of this work is, therefore, the construction of a tool turned on both ease of use and good expressiveness. At the same time, means to provide the formal representation of applications aiming at its analysis is also a great contribution.

The model proposed distinguishes intentionally the concepts of logical structuring and temporal synchronization. The logical structure of the applications facilitates its organization in chapters, sections or in any other unit. For this reason, the application becomes modular, which contributes to lessen the complexity of the scenes and to avoid the occurrence of the state explosion problem. 
The future works include the creation of mechanisms that allow the user to define in the own tool parameters of quality of service, which will be used during the execution of the application. The possibility to define alternative media objects is also an important future task.

It's important to highlight that the use of this environment integrated to the other tools being developed in the project provides a complete framework covering all the steps involved in distributed multimedia applications design: specification, verification and presentation. The easiness of the authoring model presented to the user and the use of a formal description technique to validate the applications created by him turn the environment attractive and easy to use without restricting the expressiveness of the tool.

\section{REFERENCES}

Blakowski, G. and Steinmetz, R. (1996) A Media Synchronization Survey: Reference Model, Specification, and Case Studies. IEEE Journal on Selected Areas in Communications, 14, 5-35.

Courtiat, J. P. and de Oliveira, R.C. (1996) Proving Temporal Consistency in a New Multimedia Synchronization Model. ACM Multimedia, Boston, November 1996.

Hirzalla, N.; Falchuk, B. and Karmouch, A. (1995) A Temporal Model for Interactive Multimedia Scenarios. IEEE Multimedia, Fall 1995, 24-31.

ISO/IEC DIS 13522-5. (1995) Information Technology - Coding of Multimedia and Hypermedia Information, Part 5: Support for Base-Level Interactive Applications.

ISO/IEC JTC1/SC21/WG7. (1997) Enhancements to LOTOS. Revised Working Drafts on Enhancements to LOTOS (V4), Project WI 1.21.20.2.3.

Sénac, P.; Diaz, M. and de Saqui-Sannes, P. (1994) Toward a formal specification of multimedia synchronization scenarios. Ann. Telécommun., 49, 297-314.

Sénac, P.; Willrich, R. and de Saqui-Sannes, P. (1995) Hierarchical Time Stream Petri Nets: A Model for Hypermedia Systems. Lecture Notes in Computer Science, 935, 451-470.

Soares, L. e Rodrigues, R. (1997) Autoria e Formatação Estruturada de Documentos Hipermídia com Restrições Temporais. In Proc. of III Workshop sobre Sistemas Multimídia e Hipermídia, 183-197. (In Portuguese) 